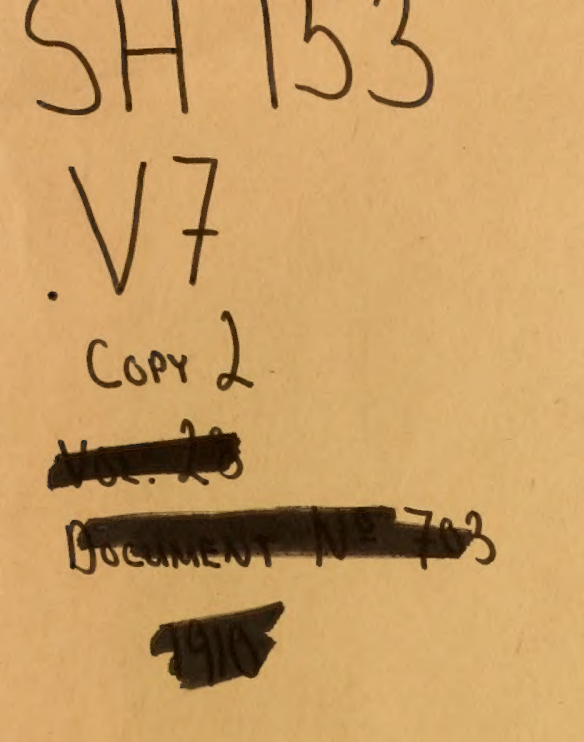




\section{A METHOD OF MEASURING FISH EGGS}

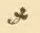

By H. von Bayer, C. E.

Architect and Engineer, United States Bureau of Fisheries

$+$

- Paper presented before the Fourth International Fishery Congress

held at Washington, U. S. A., September 22 to 26, 1908

BULLETIN OF THE BUREAU OF FISHERIES : : : : : VOL. XXVIII, P. 1009-1014

Document No. 703 : : : : : : : : : : : : : : : : : : : : Issued April, 1910 


$$
5 \times 15^{33}
$$

APR 151910

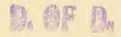

$\because \vdots$ 


\title{
A METHOD OF MEASURING FISH EGGS.
}

\author{
$*$ \\ By H. VON BAYER, C. E., \\ Architect and Engineer, United States Bureau of Fisheries.
}

*

In a well-regulated fish hatchery it becomes at times necessary to count the eggs of fishes, so as to know the quantity on hand and prepare for certain shipments of eggs as well as for the future care of the fry. The methods thus

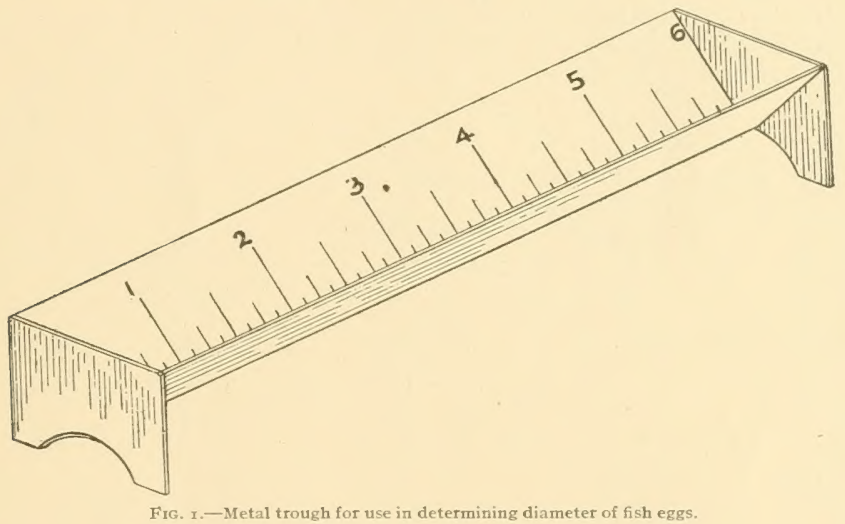

far employed have been to determine by actual count the number of eggs contained in one liquid quart measure, and then to multiply said number by the number of quarts of eggs on hand; or to weigh one liquid quart of counted eggs, next to weigh all the eggs on hand, and then by simple proportion to determine the number of all the eggs.

The new method proposed by the writer is first to determine the diameter ${ }^{a}$ of one egg, and then to enter with the value of said diameter a table or diagram

a By diameter is here understood the diameter of the egg including its surrounding matrix, if any. 
in which the corresponding number of eggs per liquid quart or other unit measure is found by inspection.

To determine the diameter of one egg of a certain species of fish, a Vshaped metal trough with scale engraved thereon is used, in which a certain number of eggs is placed one egg deep in a row, the eggs touching each other; the space occupied by the eggs is then read on the scale; this reading, when divided by the number of eggs in the trough, will give the diameter of one egg.

The accompanying table and diagram are self-explaining. They are based on a series of actual counts of eggs contained in a liquid quart measure, these counts fairly agreeing with each other and the theoretical value, and being extended by computation according to the law that solids increase as the third power of their diameters.

Example:

$\mathrm{d}=\mathrm{O} . \mathrm{I} 27^{\prime \prime}$, diameter of whitefish egg (determined).

$\mathrm{n}=33, \mathrm{o} 36$, number of whitefish eggs per quart (actually counted).

$\mathrm{d}_{1}=0 . \mathrm{I}_{4} \mathrm{O6}^{\prime \prime}$, diameter of shad egg (determined).

$\mathbf{n}_{1}=$ Number of shad eggs per quart (sought).

$\mathrm{d}^{3}: \mathrm{d}_{1}{ }^{3}=\mathrm{n}_{1}: \mathrm{n}$

$\therefore \mathrm{n}_{1}=\frac{\mathrm{d}^{3} \mathrm{n}}{\mathrm{d}_{1}{ }^{3}}$, or

$0.127^{3}: 0.1406^{3}=n_{1}: 33,036$

$\mathrm{n}_{1}=\frac{0.127^{3} \times 33,036}{0.1406^{3}}=24,345$, answer. 
METHOD OF MEASURING FISH EGGS.

TAble for Finding Number of Fish Eggs of Given Diameter per Liquid Quart.

\begin{tabular}{|c|c|c|c|c|c|c|c|}
\hline $\begin{array}{c}\text { Diame- } \\
\text { ter. }\end{array}$ & Number. & $\begin{array}{l}\text { Diame- } \\
\text { ter. }\end{array}$ & Number. & $\begin{array}{c}\text { Diame- } \\
\text { ter. }\end{array}$ & Number. & $\begin{array}{c}\text { Diame- } \\
\text { ter. }\end{array}$ & Number. \\
\hline \multirow[t]{2}{*}{$\begin{array}{l}\text { Inch. } \\
0.300\end{array}$} & 2,506 & $\begin{array}{l}\text { Inch. } \\
0.230\end{array}$ & 5.562 & $\begin{array}{l}\text { Inch. } \\
0.160\end{array}$ & I6.52I & Inch. & 92,826 \\
\hline & $\begin{array}{l}2,500 \\
2,531 \\
2,557 \\
2,583 \\
2,609\end{array}$ & & $\begin{array}{l}5.502 \\
5.635 \\
5.709 \\
5,785 \\
5.862\end{array}$ & & $\begin{array}{l}10,521 \\
16,835 \\
17,157 \\
17,487 \\
17,825\end{array}$ & & $\begin{array}{r}92,820 \\
95,990 \\
99,297 \\
102,762 \\
106,390\end{array}$ \\
\hline \multirow[t]{4}{*}{0.295} & 2,636 & 0.225 & $5.94 \mathrm{I}$ & 0.155 & 18,172 & 0.085 & I 10,190 \\
\hline & $\begin{array}{l}2,663 \\
2,690\end{array}$ & & $\begin{array}{l}6.021 \\
6,102\end{array}$ & & $\begin{array}{l}18,528 \\
18,894\end{array}$ & & $\begin{array}{l}114,172 \\
118,346\end{array}$ \\
\hline & 2,718 & & 6,185 & & 19.270 & & 122,730 \\
\hline & 2,746 & & 6,269 & 0.151 & I9.655 & & 127,333 \\
\hline \multirow[t]{4}{*}{0.290} & 2. 775 & 0.220 & 6.355 & 0.150 & 20,050 & 0.080 & 132,170 \\
\hline & $\begin{array}{l}2,804 \\
2,833\end{array}$ & & $\begin{array}{l}6,442 \\
6,531\end{array}$ & & $\begin{array}{l}20,456 \\
20,874\end{array}$ & & $\begin{array}{l}137.251 \\
142,600\end{array}$ \\
\hline & 2,863 & & 6.622 & & 21,303 & & 148,220 \\
\hline & 2,893 & & 6.715 & & 21,744 & & I 54,155 \\
\hline \multirow[t]{4}{*}{0.285} & 2,923 & 0.215 & 6.809 & o. $x 45$ & 22,197 & 0.075 & 160.400 \\
\hline & $\begin{array}{l}2,954 \\
2,985\end{array}$ & & 6.905 & & 22,662 & & I 66.995 \\
\hline & $\begin{array}{l}2.985 \\
3.017\end{array}$ & & $\begin{array}{l}7,002 \\
7,102\end{array}$ & & & & 173,950 \\
\hline & $\begin{array}{l}3.017 \\
3.050\end{array}$ & & $\begin{array}{l}7.102 \\
7.204\end{array}$ & & $\begin{array}{l}23,033 \\
24,140\end{array}$ & & $\begin{array}{l}181,300 \\
189,070\end{array}$ \\
\hline \multirow{5}{*}{0.280} & 3.083 & 0.210 & 7.307 & 0.540 & 24.661 & 0.070 & 197,290 \\
\hline & 3.116 & & 7.412 & & 25,197 & & 205,992 \\
\hline & 3.150 & & 7.520 & & 25.748 & & 215,204 \\
\hline & 3.184 & & 7.629 & & 26.316 & & 224,995 \\
\hline & 3,219 & & $7.74 \mathrm{I}$ & & $26,90 \mathrm{I}$ & & 235.377 \\
\hline \multirow[t]{4}{*}{0.275} & 3.254 & 0.205 & 7.855 & 0.135 & 27.504 & 0.065 & 246,410 \\
\hline & $\begin{array}{l}3.290 \\
3,326\end{array}$ & & $\begin{array}{l}7.971 \\
8,089\end{array}$ & & $\begin{array}{l}28,125 \\
28,764\end{array}$ & & $\begin{array}{l}258,141 \\
270,631\end{array}$ \\
\hline & 3.363 & & 8,2 ro & & 29,422 & & 283.936 \\
\hline & 3.400 & $0.20 \mathrm{I}$ & 8.333 & & 30, IOI & & $298, x_{32}$ \\
\hline \multirow[t]{4}{*}{0.270} & 3.438 & 0.200 & 8,459 & 0.130 & $30,80 x$ & 0.060 & 313,289 \\
\hline & $\begin{array}{l}3.476 \\
3.515\end{array}$ & & $\begin{array}{l}8,587 \\
8,717\end{array}$ & & $\begin{array}{l}31,523 \\
32,268\end{array}$ & & $\begin{array}{l}329,490 \\
346,828\end{array}$ \\
\hline & 3.555 & & $8,85 \mathrm{x}$ & & 33.036 & & 365,405 \\
\hline & 3.595 & & 8.987 & & 33,829 & & 385.331 \\
\hline \multirow[t]{4}{*}{0.265} & 3.636 & o. 195 & 9,126 & 0.125 & 34,647 & 0.055 & 406,733 \\
\hline & 3.677 & & 9,268 & & 35,492 & & 429.750 \\
\hline & 3. 719 & & 9.413 & & 36,364 & & 454.539 \\
\hline & 3.762 & & $9.56 I$ & & 37,265 & & $48 \mathrm{r}, 270$ \\
\hline \multirow{5}{*}{0.260} & $\begin{array}{l}3.806 \\
3.850\end{array}$ & 0.190 & $\begin{array}{l}9,712 \\
9,866\end{array}$ & 0.120 & $\begin{array}{l}38, \times 98 \\
39,16 \mathrm{r}\end{array}$ & 0.050 & $\begin{array}{l}510,139 \\
541\end{array}$ \\
\hline & $\begin{array}{l}3.850 \\
3.895\end{array}$ & & 10,023 & 0.1200 & $\begin{array}{l}39,16 \mathrm{I} \\
40,156\end{array}$ & 0.050 & $\begin{array}{l}54 x, 362 \\
575,173\end{array}$ \\
\hline & 3.940 & & 10,184 & & $41, x 86$ & & $61 \mathrm{x}, 893$ \\
\hline & 3. 986 & & 10,348 & & 42,251 & & $65 \mathrm{x}, 776$ \\
\hline & 4.033 & & 10,516 & & 43,354 & & 695,223 \\
\hline \multirow[t]{4}{*}{0.255} & $4.08 \mathrm{I}$ & 0.185 & 10.688 & 0.115 & 44.494 & 0.045 & 742,613 \\
\hline & 4. 129 & & 10,863 & & 45,676 & & 794.400 \\
\hline & $4, \times 78$ & & II, 042 & & 46,899 & & $85 \mathrm{r}$, I 28 \\
\hline & 4,228 & & II, 225 & & 48,166 & & 913.380 \\
\hline \multirow{6}{*}{0.250} & 4.279 & & $\mathrm{II}, 4 \mathrm{I} 2$ & & $49 \cdot 4^{80}$ & & $98 I, 852$ \\
\hline & 4.331 & 0.180 & I 1.603 & 0.110 & $50,84 x$ & 0.040 & $x, 057,350$ \\
\hline & 4. 383 & & I I, 799 & & 52,254 & & $1,140,780$ \\
\hline & 4.436 & & I1, 999 & & 53,720 & & $1,233,250$ \\
\hline & 4. 490 & & 12,203 & & 55,239 & & I, 335.960 \\
\hline & 4.545 & & 12,412 & & 56,817 & & I, 450,406 \\
\hline \multirow[t]{5}{*}{0.245} & 4. $60 \mathrm{r}$ & 0.175 & 12.627 & 0.105 & 58,456 & 0.035 & I. 578,320 \\
\hline & 4.658 & & 12,846 & & 60,159 & & $\mathrm{I}, 72 \mathrm{I}, 630$ \\
\hline & 4.716 & & 13.069 & & 61,925 & & $1,88_{3}, 020$ \\
\hline & 4. 776 & & I3. 298 & & 63.766 & & $2,065,130$ \\
\hline & 4.835 & & 13.533 & o. ror & 65,680 & & $2,271,500$ \\
\hline \multirow[t]{5}{*}{0.240} & 4.895 & 0.170 & 13,774 & 0. 100 & 67,670 & 0.030 & $2,506,310$ \\
\hline & 4.956 & & 14,020 & & $69.74 I$ & & \\
\hline & 5.019 & & 14.272 & & 71,899 & & \\
\hline & 5,083 & & 14.529 & & $\begin{array}{l}74,146 \\
76,486\end{array}$ & & \\
\hline & $\begin{array}{l}5,148 \\
5,214\end{array}$ & 0.165 & $\begin{array}{l}\text { I } 4,793 \\
\text { I5, } 064\end{array}$ & 0.095 & $\begin{array}{l}70,400 \\
78,927\end{array}$ & & \\
\hline \multirow{4}{*}{0.235} & $\begin{array}{l}5,214 \\
5,281\end{array}$ & & $\begin{array}{l}15,004 \\
15,341\end{array}$ & 0.095 & $8 x, 473$ & & \\
\hline & 5.350 & & I5. 625 & & 84,130 & & \\
\hline & 5.419 & & $\begin{array}{l}15.916 \\
16.215\end{array}$ & & $\begin{array}{l}86,904 \\
89.800\end{array}$ & & \\
\hline & 5.490 & & & & & & \\
\hline
\end{tabular}

\section{CONVERSION TABLE.}

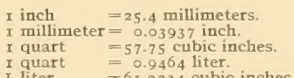

$\begin{array}{ll}\text { I quart } & =0.9464 \text { liter. } \\ \text { I liter } & =61.0234 \text { cubic inches. }\end{array}$

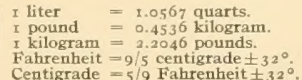

I pound $=0.4536$ kilogra

Fahrenheit $=9 / 5$ centigrade $\pm 32^{\circ}$. 


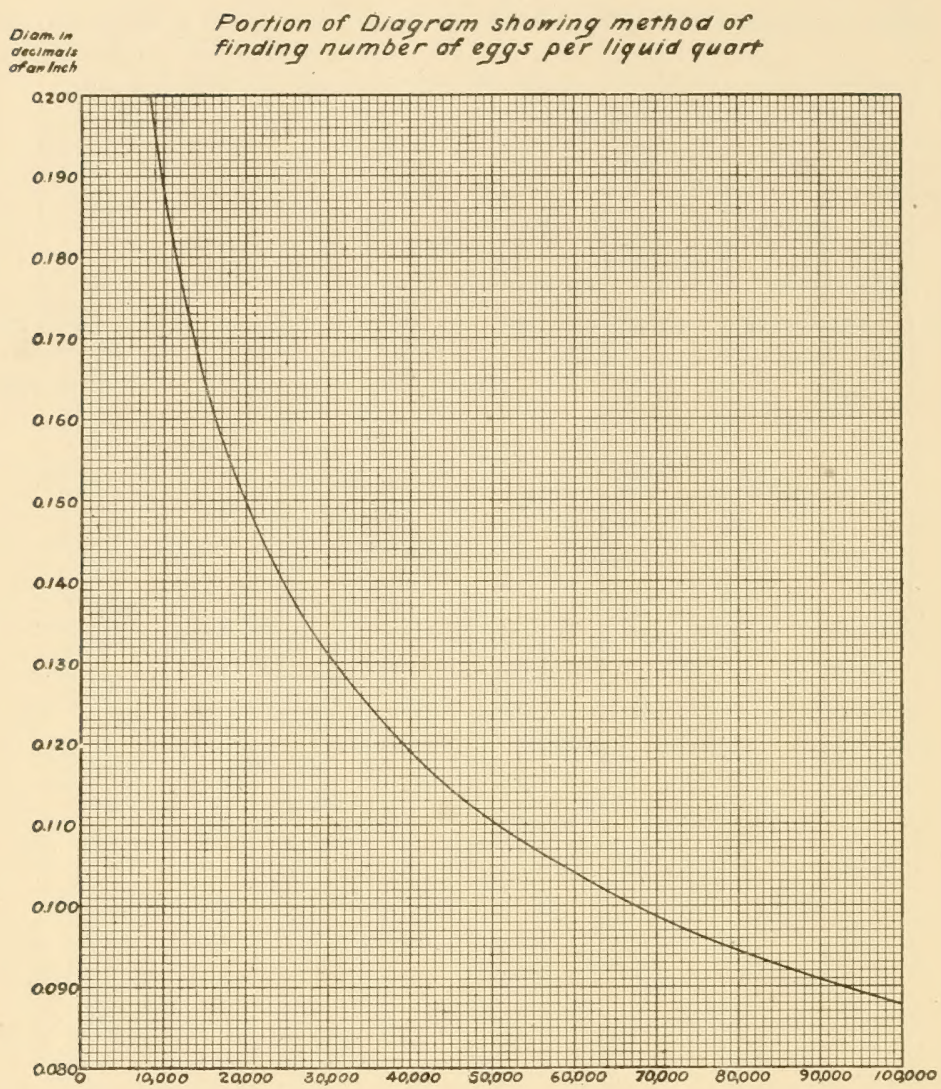

Directions: Find the line on the left margin corresponding to the given diameter; follow said line to the right until it intersects the curve; from this intersection proceed at right angles to the lower marginal line of figures and there read the required number of eggs per quart.

If diameter is given in millimeters multiply by 0.03937 to reduce to inches. 



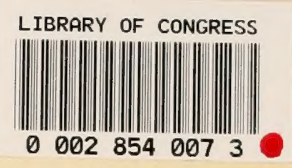





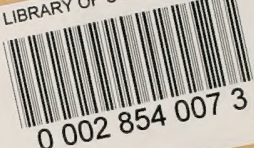

Hollinger Corp.

pH 8.5 